\title{
Análisis de las metodologías pedagógicas empleadas en el área de tecnología e informática para el fortalecimiento de las competencias digitales
}

\author{
Analysis of the pedagogical methodologies used in the \\ technology area to strengthen digital competencies
}

LILIANA BELTRÁN CHIRIVI

Corporación universitaria Minuto de Dios - UNIMINUTO, Bogota, Colombia(liliana.beltran-c@ uniminuto.edu.co)(https://orcid.org/0000-0001-6249-6863)

ADRIANA CASTRO CAMELO

Corporación universitaria Minuto de Dios - UNIMINUTO, Bogota, Colombia(adriana.castro@ uniminuto.edu)(https://orcid.org/OOOO-0002-0779-7740) MARISOL ESPERANZA CIPAGAUTA MOYANO

Corporación universitaria Minuto de Dios - UNIMINUTO, Bogota, Colombia(mcipagauta@ uniminuto.edu)(https://orcid.org/0000-0002-1378-8824)

\section{RESUMEN}

El presente artículo da cuenta de una investigación realizada en una comunidad educativa de bajos recursos, con un alto índice de vulnerabilidad y necesidades básicas insatisfechas, como la falta de oportunidades laborales, desatención en salud y un alto índice de desescolarización. Desde estas condiciones surge la necesidad de facilitar herramientas educativas que enfoquen al estudiante de educación básica y media a fortalecer las competencias digitales y comunicacionales mediante la creación de contenidos, la seguridad informática y la resolución de problemas. El estudio se realizó desde un enfoque metodológico cualitativo, que permitió orientar el proyecto dando respuesta a los objetivos propuestos, empleando diversos instrumentos de recolección de datos para el posterior análisis de las metodologías pedagógicas empleadas.

\section{ABSTRACT}

This article is about an investigation carried out in a low-income educational community, with a high rate of vulnerability and unsatisfied basic needs, such as lack of job opportunities, inattention to health, and a high rate of out-of-school attendance. From these conditions arises the need to provide educational tools that 
focus the student of basic and secondary education to strengthen digital and communication skills through content creation, computer security and problem solving. The study was carried out from a qualitative methodological approach, which allowed guiding the project by responding to the proposed objectives, using various data collection instruments for the subsequent analysis of the pedagogical methodologies used.

\section{PALABRAS CLAVE / KEY WORDS}

Tecnología; pedagogía; competencia digital; informática./Technology; pedagogy; digital competence; computing.

\section{INTRODUCCIÓN}

Las metodologías educativas que desarrollan los docentes en las aulas de clase permiten que el proceso de enseñanza aprendizaje se fortalezca, se conviertan en herramientas de apoyo a las necesidades educativas y se mejore la comprensión de los conceptos y contenidos en los cuales presentan dificultades académicas. Sin embargo, en tecnología e informática, estas metodologías se convierten en orientaciones prácticas para el desarrollo personal de cada uno de los estudiantes, siendo un poco más flexible en comparación con las diferentes áreas de aprendizaje.

De igual forma, las temáticas establecidas en las aulas de clase, son solamente para los estudiantes una serie de actividades que generan unas calificaciones, por eso se ven obligados a cumplir, pero no ven estas actividades como un enfoque personal que permita vivenciar fuera de las instituciones educativas, siendo esto una limitante para obtener los resultados esperados por los diferentes docentes que orientan el área de tecnología e informática.

Por lo anterior, la investigación realizada presenta un análisis detallado de las metodologías que establecen los docentes de tecnología e informática en el aula de clase del colegio Técnico Tomás Rueda Vargas; y cómo, a partir de ellas, se puede fomentar las competencias digitales en los estudiantes de los grados octavo, cursos que facilitan generar proyectos académicos con seguimiento continuo y progresivo en lo que se evidencien las diferentes estrategias y metodologías que desarrolla la profesión docente. Bunge (2017) refiere que las competencias digitales son "un proceso indispensable en la labor docente y en la educación en general" (p. 146).

Es de resaltar que este estudio busca una transformación a fondo de la manera de enseñar en las aulas de clase de tecnología e informática que permita responder al avance tecnológico que se lleva a cabo a nivel mundial y generar desde las aulas de clase un modelo de escuela digno, capaz de generar una propuesta que apoye el desarrollo tecnológico del país y especialmente a las familias y el entorno social de los mismos estudiantes. 
Aunque la metodología utilizada, es de carácter cualitativo, se emplearon tres instrumentos, que no solo permitieron observar las características de las técnicas empleadas por los docentes de tecnología e informática, sino además permiten establecer un informe cuantitativo, con la puesta en marcha del cuestionario docente, la entrevista y el formato de recolección de datos no participante, que dejaron establecer la dificultad de fortalecer las competencias digitales en los colegios públicos, debido a la falta misma de interés por parte de los estudiantes dentro del sistema educativo.

Finalmente, este estudio cualitativo evidenciará las herramientas y metodologías utilizadas en el aula de clase, permitiendo sugerir y fortalecer las metodologías por los docentes, con la finalidad de enfocar a los estudiantes a mejorar las competencias digitales, orientando a los estudiantes a ver la tecnología como una herramienta laboral y por qué no, profesional. Castilla (2018) dice que las TIC "son las que permiten gestionar y transformar datos que pueden llevarse a todos lados y ser administrados para tener un acceso fácil que lleva a la producción y transferencia de nuevo conocimiento" (p. 23), por lo que las TIC también son una forma de acceder fácilmente a la información y permite compartirla en cualquier lugar del mundo.

\section{ANTECEDENTES}

Los docentes en los últimos años han tenido que actualizar sus conocimientos y especialmente las competencias digitales, debido al auge que ha tenido la tecnología a nivel mundial y especialmente a nivel educativo. Las metodologías también han sido expuestas a estos cambios de manera más eficiente, porque se han condensado en el aprendizaje del estudiante, en este sentido Hernández (2017) de la Universidad San Ignacio de Loyola, a través de su investigación "Impacto de las TIC en la educación: retos y perspectivas" refiere, cómo la innovación en la educación es un estimulante para la creación de nuevos conocimientos y describe a su vez la importancia de la comunidad educativa para transformar el proceso de enseñanza aprendizaje en el ámbito educativo.

Sin embargo, el objetivo principal es convocar a nuevas innovaciones de las tecnologías de la información y las comunicaciones para convertirla en sociedad del conocimiento, para que cada agente educativo (docente-estudiante) transforme la educación a través de nuevas prácticas pedagógicas y tecnológicas desarrolladas dentro del aula de clase, estableciendo de este modo, un paso gigante en la transformación mundial de la educación y es allí, donde ésta investigación, desea analizar las metodologías que emplean los docentes de aula, en especial los docentes de tecnología e informática del colegio Técnico Tomás Rueda Vargas, para evidenciar, sugerir o transformar también, metodologías innovadoras, capaces de convertir la sociedad en una sociedad de conocimiento, creativa y eficaz.

En la investigación realizada por Campos (2017), sobre “La alfabetización tecnológica: de la informática al desarrollo de competencias tecnológicas", realizada en la Universidad Austral de Chile, refuta como el sistema educativo no colabora con el aumento de la competitividad y productividad de la población, debido a que no facilita el desarrollo de tales habilidades y competencias. Dicha investigación utilizó una metodología de aprendizaje basada en proyectos con uso de Tecnologías de la Información y la Comunicación (TIC), donde 
tomo una muestra de seis profesores y 95 estudiantes de cinco municipios de Colombia, analizando de manera cualitativa la información, por medio de un proceso de triangulación que contempla categorías preestablecidas y emergentes. Los resultados revelan que la estrategia de aprendizaje es significativa en los diferentes roles de la comunidad educativa y específicamente en las competencias tecnológicas. Esta investigación concluye que la estrategia de aprendizaje es fundamental para la alfabetización tecnológica, conclusión que afianza la presente investigación, al considerar que las metodologías tecnológicas fortalecen las competencias digitales.

Guevara (2017), líder de la academia de proyectos de la carrera de ingeniería en tecnologías de manufactura de la ciudad de México, en su investigación "Prototipos tecnológicos exitosos e innovadores de bajo presupuesto realizados por los alumnos en universidades públicas" buscó la vinculación de proyectos elaborados por los estudiantes con bajos recursos, junto con el departamento de vinculación. Esta investigación fue ganadora del primer y segundo puesto en el "VII Premio Concyteg a la Innovación Tecnológica" Guanajuato (2014), en el “Foro Internacional Sinnco" y en el "5to Congreso Nacional de Manufactura", (2014-2015); propone una metodología lúdica para lograr la vinculación de estos prototipo, con el objetivo de fomentar el crecimiento económico y desarrollo de capacidades, como reto global a los que se enfrentan los estudiantes en la actualidad. Los resultados se dieron a conocer por diferentes medios, de tal forma que sirvieran de incentivo para otros estudiantes y así generar el interés en la vinculación de sus proyectos.

Alonso (2017), de la universidad de Buenos Aires, en su investigación titulada “¿Hacia una nueva definición de utilidad del conocimiento científico?”, describe el desarrollo de la estrategia de análisis cualitativo, provenientes de la primera etapa de trabajo de campo, su objetivo principal es comprender cuáles mecanismos llevan adelante los investigadores en lo que respecta a instrumentos de investigación, orientado a los proyectos de desarrollo Tecnológico y Social (PDTS), proyectos que utiliza una metodología orientada a introducir elementos novedosos en lo que respecta a las competencias académicos, como también a la vinculación con actores no académicos, y es precisamente alli donde esta investigación aporta a la actual, ya que hace un guía a la utilización de instrumentos investigativos y enfatiza en las metodologías que se emplean a nivel tecnológico, como al aporte que hace al fortalecimiento de las competencias digitales.

En la investigación desarrollada por Martínez (2011), denominada: “El método de estudio de caso estrategia metodológica de la investigación científica” elaborada en la Universidad del Norte, se evidencia la importancia de la metodología, los procedimientos y elementos utilizados en el aula de clase, demostrando tanto las características claves como el valor y la creatividad. El propósito de esta investigación es el beneficio y la utilidad práctica de las metodologías empleadas a través del método de casos, método que se hace natural en el aula de clase, donde se evidencia las dificultades cotidianas que presentan los estudiantes y lo cual aporta significativamente al presente trabajo.

\section{MARCO TEÓRICO}

Actualmente, se evidencia un gran auge tecnológico debido a la crisis sanitaria que se 
vive, teniendo que convertir la tecnología en una herramienta primordial y cotidiana en la vida del ser humano; gracias a la evolución de las herramientas tecnológicas, es fácil llevar a cabo una clase virtual, donde no solo se trasmiten saberes, si no que adicionalmente se cuenta con la interactividad auditiva y visual entre los docentes y los estudiantes. Para los trámites educativos, como certificados, boletines y demás documentos que anteriormente debía solicitarse cita para expedirlos, ahora esta aun solo clic y es tanto la evolución virtual que el mundo no se detuvo, gracias a esta misma evolución.

Teniendo en cuenta el acelerado avance tecnológico, las instituciones educativas, llámese centros educativos, universidades y demás, han enfocado su proceso tecnológico a metodologías tradicionales que conllevan a la elaboración de actividades como evaluación y/o resultado final de una serie de conceptos, es así como en Gran Bretaña un grupo de niñas en el proceso de enseñanza aprendizaje diseñaron diferentes prototipos para dar respuesta a las necesidades de la comunidad; British Council (2018), inició un concurso entre estudiantes de 12 a 14 años de varios países, donde más de 250 estudiantes participaron en el proceso y desarrollaron diversos prototipos y/o herramientas, además de la finalidad del concurso se determinó la importancia del acompañamiento docente y especialmente a las metodologías empleadas por cada uno de ellos, que si bien daba respuesta a la actividad, no eran resultado de las metodologías empleadas en clase, entre algunos de los ejemplos de los proyectos presentados se encuentran dispositivos para buenos hábitos alimenticios, ayudar a personas con Alzheimer y para hacer ejercicio, entre otros.

La importancia de las metodologías empleadas en el sistema educativo y específicamente en el área de tecnología contribuyen a la formación tecnológica desde básica secundaria, hasta finalizar los estudios de educación media, como una herramienta educativa y laboral que pueden emplear al egresar de las instituciones convirtiéndose en competencias indispensables para el desarrollo personal y especialmente en una población cuyos recursos económicos, termina siendo una situación muy compleja. Estas metodologías están guiadas al proceso cualitativo, cuantitativo o mixto, donde afianza el conocimiento en toda investigación, donde al implementar cualquiera de estos tipos de metodologías, hacen que el investigador tenga una visión más clara al obtener los resultados y cualificar o cuantificar los mismos.

\section{METODOLOGÍAS EDUCATIVAS}

En la actualidad el mundo está cambiando, especialmente en el contexto tecnológico y por ello los docentes buscan nuevas metodologías y/o métodos de enseñanza - aprendizaje para utilizarlos y poder reinventar las prácticas docentes, motivando a los estudiantes a través de métodos novedosos capaces, no solo de trasmitir conocimientos, sino a demás cautivar al estudiante y potencializar sus capacidades y competencias.

Algunos de estos métodos que afianzan y potencian las metodologías utilizadas por los docentes son:

Aprendizaje basado en Proyectos (ABP): el Aprendizaje Basado en Proyectos permite adquirir conocimientos y competencias a través de la elaboración de proyectos educativos, 
que ofrecen repuesta a las necesidades cotidianas de los estudiantes, garantizando procesos didácticos y prácticos que desarrollan competencias básicas en la comunicación, resolución de problemas, pensamiento crítico y colaborativo.

Flipped Classroom (Aula Invertida): esta metodología permite al estudiante alistar, analizar y preparar los implementos o recursos o educativos en casa para minimizar contratiempos y maximizar el tiempo en clase, una vez esta se desarrolle.

Aprendizaje Cooperativo: esta metodología desarrolla en el estudiante la competencia de trabajo en grupo, desarrollando diferentes actividades que permite los miembros del grupo realicen con éxito las tareas establecidas apoyándose en el trabajo de los demás.

Gamificación: esta metodología permite potenciar la motivación, concentración, esfuerzo y valores positivos a través de videojuegos y dinámicas de juego implementadas en el aula de clase.

Design Thinking (Pensamiento de Diseño): el pensamiento de diseño como metodología, cumple la funcionalidad de resolver problemas e identificarlos individualmente para generar ideas y soluciones creativas en cada uno de los estudiantes.

Aprendizaje Basadoen el Pensamiento (Thinking Based E-learning): la metodología permite desarrollar destrezas del pensamiento, contextualizando, analizando y argumentando el entorno en que se desenvuelve, haciendo propio el conocimiento y no memorístico, basado igualmente por el pensamiento (TBL).

Aprendizaje Basado en Competencias: esta metodología se basa en la demostración de los resultados de aprendizaje cuya finalidad en el proceso de aprendizaje en el estudiante está enfocado a dominar la capacidad que se tiene para desarrollar dicha actividad.

\section{CARACTERÍSTICAS DE LA DIDÁCTICA TECNOLÓGICA}

En la didáctica tecnológica se debe contemplar características específicas, no solo de los roles que cumplen los docentes y estudiantes, sino además de factores y/o procesos que intervienen, entre ellos se pueden observar:

\begin{tabular}{|l|l|}
\hline \multicolumn{2}{l}{ Tabla 1. Caracteristicas de las didácticas tecnológicas } \\
Factores & \multicolumn{1}{c|}{ Características } \\
\hline Docente & $\begin{array}{l}\text { Dominio del tema, flexibilidad, implementación de materiales pedagógi- } \\
\text { cos, organización de los espacios, teoría y práctica en el proceso de ense- } \\
\text { ñanza }\end{array}$ \\
Fuente: elaboración propia.
\end{tabular}




\begin{tabular}{|c|c|}
\hline Factores & Caracteristicas \\
\hline Estudiante & $\begin{array}{l}\text { Objeto de la enseñanza, persona activa, responsabilidad en la elaboración } \\
\text { de las tareas asignadas, resolución de problemas con fundamentos sólidos, } \\
\text { aprovechar lo positivo de las distintas teorías del aprendizaje, utilización de } \\
\text { herramientas tecnológicas tanto individual como grupal. }\end{array}$ \\
\hline Concepción del aprendizaje & $\begin{array}{l}\text { En esta didáctica predomina el resultado esperado y no el proceso } \\
\text { sugerido, reflexiones que provienen de un constructo teórico ampliamente } \\
\text { investigado, adopta un papel central en el proceso de enseñanza y } \\
\text { aprendizaje. }\end{array}$ \\
\hline Instrumentales básicos & $\begin{array}{l}\text { Observación, lectura, escritura, inteligencia y otras capacidades, sintetizar } \\
\text { los nuevos conocimientos e integrarlos con los saberes previos para lograr } \\
\text { su “apropiación”, experiencia, orientación, recursos didácticos, comprender } \\
\text { la nueva información. }\end{array}$ \\
\hline Objetivo del aprendizaje & $\begin{array}{l}\text { Es el punto de partida de la didáctica, ya que esta es la que define el fin de la } \\
\text { actividad. Retención a largo plazo de la información y de los conocimientos } \\
\text { asociados que se hayan elaborado. La transferencia del conocimiento a } \\
\text { nuevas situaciones para resolver con su concurso las preguntas y problemas } \\
\text { que se planteen. }\end{array}$ \\
\hline Contenidos & $\begin{array}{l}\text { Aunque hace parte del proceso, no son fundamentales dentro del mismo, } \\
\text { ya que pueden cambiar constantemente. }\end{array}$ \\
\hline Instrumentos básicos & $\begin{array}{l}\text { Realizan múltiples operaciones cognitivas que contribuyen a lograr el de- } \\
\text { sarrollo de sus estructuras mentales y de sus esquemas de conocimiento, } \\
\text { receptividad, Reflexibilidad, creatividad, expresividad simbólica, expresivi- } \\
\text { dad práctica. }\end{array}$ \\
\hline Actividad & $\begin{array}{l}\text { Esta es la diferente técnica utilizada en el momento de la clase y puede ser } \\
\text { variada de acuerdo a la motivación que implemente el docente. }\end{array}$ \\
\hline Actividades memorísticas & Permiten desarrollar procesos cognitivos lógicos \\
\hline Actividades reproductivas & $\begin{array}{l}\text { Pretenden la memorización y el recuerdo de una información determina- } \\
\text { da. }\end{array}$ \\
\hline Actividades comprensivas & $\begin{array}{l}\text { Pretenden la construcción o la reconstrucción del significado de la infor- } \\
\text { mación con la que se trabaja, revisión del conocimiento a partir del análisis } \\
\text { crítico, debate y la argumentación. }\end{array}$ \\
\hline Evaluación & $\begin{array}{l}\text { Es la encargada de verificar o comprobar que se ha cumplido con la fina- } \\
\text { lidad del proceso, aprendizaje autorregulado y disposición para aprender, } \\
\text { fuente más importante de los mensajes que el profesor proporciona con } \\
\text { su enseñanza. }\end{array}$ \\
\hline
\end{tabular}

\section{COMPETENCIAS DIGITALES}

El fortalecimiento de las competencias digitales, permiten un continuo avance tecnológico, 
centrado en los esfuerzos que realiza el ser humano, acoplando la tecnología al ritmo de vida, ya sea de forma diacrónica o sincrónica, manipulando así una serie de elementos que abarca el entorno social y natural.

Sin embargo, ¿qué tan importante es la metodología tecnológica para fortalecer las competencias digitales?, ¿hasta qué punto estas metodologías son positivos o negativas en la vida del ser humano?, ¿las competencias digitales vistas a nivel educativo satisface las necesidades más íntimas y vanas necesarias?, o ¿es simplemente una competencia educativa para complementar un tema en clase? Las generaciones actuales están creciendo de la mano de la tecnología, siendo convenientes para el entorno social que se vive hoy en día y es por esta razón que no se discierne entre lo individual y general, es decir las competencias digitales se han vuelto indispensable para todos, que no se alcanza a comprender que dichas competencias son necesarias para el avance tecnológico, donde el ser humano no debe estar al servicio de la tecnología. Si no, al contrario, como debería ser.

Dashner (2015), por otro lado, cita que "la tecnología puede usarse para muchas cosas buenas, pero, al final, somos esclavos de la naturaleza y los elementos, mientras las metodologías tienden a ser resultado de simples experimentos" (p. 32).

Por otra parte, se debe tener en cuenta las implicaciones que tiene la metodología educativa enfocada al fortalecimiento de las competencias digitales a nivel cultural, donde no se sabe a manera cierta o incierta si la cultura avanza o por el contrario retrocede, cuando algunos docentes utilizan una y otra vez las mismas metodologías tradicionales se convierten entonces en algo insignificante en la vida cotidiana, dejando entre dicho su importancia en la cultura actual, generando expresiones como la mencionada por Giner, (2008) quien afirma que “la tecno cultura no significa el naufragio de la cultura, solo su reestructura ción y la mudanza relativa de su lenguaje, consecuencia de una mala metodología” (p.105).

De igual forma las competencias digitales en el siglo XXI son esenciales en el trabajo cotidiano, el aprendizaje y participación activa, según Ocaña, Valenzuela y Morillo (2019) las competencias digitales "deben adaptarse; con lo cual todo aquel partícipe que se oriente a este mundo deberá manejar los códigos cada vez más complejos, pero muy necesarios" (p. 63) y más aún cuando el entorno social se ve amenazado por la emergencia sanitaria que impiden el normal desarrollo laboral físico y se deben implementar estrategias virtuales que contemplen los mismos objetivos, pero de manera diferente, viendo así entonces como las personas de mayor edad, no han sido capacitadas correctamente en las diferentes competencias digitales, como se observa obviamente en la población joven actual.

No obstante, al hablar de las competencias digitales es importante menciona los cinco pilares de la competencia digital docente, que esta incorpora para un mejor proceso de innovación, comunicación y aprendizaje, entre estos pilares se encuentra la informatización y alfabetización informacional; esta competencia identifica el saber equiparar, situar, rescatar, recopilar, organizar y considerar la información digital, por otra parte la segunda competencia es la comunicación y elaboración, en esta aptitud el docente debe transmitir en entornos digitales recursos y herramientas que permitan interactuar y participar en comunidades y redes.

La tercera competencia está guiada a la creación de contenido digital, enfocada a fomentar la creatividad y reelaboración de contenidos que faciliten el aprendizaje y genere nuevos conocimientos, aplicando los diversos derechos de propiedad intelectual. Actualmente es 
considerada una de las competencias básicas en la educación, donde el estudiante a través de la creatividad reconstruye desde sus propios conocimientos, nuevos conceptos y los aplica a su diario vivir.

Por su parte, la cuarta competencia; la competencia digital eficaz, permite proteger datos personales e identidades digitales para un uso sostenible y seguro; mientras la quinta competencia está enfocada a la resolución de problemas, donde la juventud hoy en día, evoluciona a nivel digital, sin embargo, al obtener diversos recursos digitales debe tomar las mejores decisiones para utilizar el dispositivo, recurso y/o herramienta digital más apropiada y de esta manera resolver dificultades cotidianas o problemas de uso técnico a que se vea enfrentado, únicamente con la finalidad de resolver problemas que permitan desarrollar la competencia adecuada.

En consecuencia, con ayuda de esta competencia genera nuevos procesos cognitivos que permiten reorganizar las diferentes estructuras mentales y posteriormente facilitar la comprensión y solución de cualquier inconveniente, problema o situación a la que se vea enfrentado no solo educativa, emocional sino cotidianamente.

\section{METODOLOGÍA}

El colegio Tomás Rueda Vargas se encuentra localizado en la ciudad de Bogotá D.C, en la localidad de San Cristóbal sur, es un megacolegio con más de 2500 estudiantes, su contexto educativo es de carácter público de la Secretaría de Educación de Bogotá, los docentes de la sede A, que orientan los procesos de los grados octavo en la asignatura de tecnología e informática. De igual manera, se desarrolla una muestra probabilística de estudiantes, es decir, todos los estudiantes de los cursos mencionados (801-804), tienen la misma posibilidad de ser elegidos, sin embargo, solo 20 estudiantes de estos dos grupos, son seleccionados aleatoriamente y participa como muestra del proyecto, esta muestra tiene la ventaja de medir el tamaño del error y hacer las correcciones pertinentes.

\begin{tabular}{|c|c|}
\hline \multicolumn{2}{|r|}{ Docentes Entrevistados } \\
\hline Docentes & Perfil profesional y experiencia académica \\
\hline $\begin{array}{l}\text { Docente } 1 . \\
\text {-Licenciado en Tecnología e informática } \\
\text {-Tiempo de dedicación: Medio Tiempo } \\
\text {-Tiempo en la institución: } 8 \text { años }\end{array}$ & $\begin{array}{l}\text { Licenciado en Tecnología e informática de la Fundación univer- } \\
\text { sitaria Unipanamericana, ubicada en Bogotá. Especialista en } \\
\text { Computación para la docencia a distancia. Diplomado en Inves- } \\
\text { tigación y en Docencia Virtual. Un total de } 23 \text { años en diferentes } \\
\text { instituciones educativas. }\end{array}$ \\
\hline $\begin{array}{l}\text { Docente } 2 . \\
\text { - Licenciado en Informática } \\
\text {-Tiempo de dedicación: Completo } \\
\text {-Tiempo en la institución: } 3 \text { años }\end{array}$ & $\begin{array}{l}\text { Licenciado en Informática de la Universidad Fundación Univer- } \\
\text { sitaria Católica } \\
\text { - Unicatólica. } \\
\text { Docente hace } 6 \text { años }\end{array}$ \\
\hline
\end{tabular}




\section{Tabla 2. Caracteristicas de los profesores entrevistados}

\section{Docentes Entrevistados}

\begin{tabular}{|c|c|}
\hline Docentes & Perfil profesional y experiencia académica \\
\hline $\begin{array}{l}\text { Docente } 3 . \\
\text {-Magister en educación virtual } \\
\text { - Licenciada en Educación } \\
\text {-Tiempo de dedicación: Completo } \\
\text {-Tiempo en la institución: } 2 \text { años }\end{array}$ & $\begin{array}{l}\text { Magíster en Educación Virtual de la universidad cooperativa de } \\
\text { Colombia } \\
\text { Licenciada en educación de la Universidad Santander } \\
\text { Docente hace } 16 \text { años }\end{array}$ \\
\hline $\begin{array}{l}\text { Docente } 4 . \\
\text {-Magister en Tecnologías digitales } \\
\text { aplicada a la educación } \\
\text {-Especialista en aplicación de Tic para la } \\
\text { enseñanza } \\
\text { - Licenciada en Biología y Química } \\
\text {-Tiempo de dedicación: Completo } \\
\text {-Tiempo en la institución: } 5 \text { años }\end{array}$ & $\begin{array}{l}\text { Magíster en Tecnologías digitales aplicadas a la educación de la } \\
\text { Universidad de Santander Udes } \\
\text { Especialista en aplicación de Tic para la enseñanza de la Univer- } \\
\text { sidad Santander Udes } \\
\text { Licenciad a en Biología y Química de la Universidad Tecnológica } \\
\text { del Choco "Diego Luis Córdoba” } \\
\text { Docente hace } 17 \text { años }\end{array}$ \\
\hline $\begin{array}{l}\text { Docente } 5 . \\
\text {-Magister en Educación } \\
\text { - Licenciada en Educación Básica con én- } \\
\text { fasis en Informática Educativa } \\
\text {-Tiempo de dedicación: Completo } \\
\text {-Tiempo en la institución: } 1 \text { año }\end{array}$ & $\begin{array}{l}\text { Magíster en Educación de la Corporación Universitaria Minuto } \\
\text { de Dios UNIMINUTO } \\
\text { Licenciada en Educación Básica con énfasis en Informática Edu- } \\
\text { cativa de la Fundación Universitaria Panamericana } \\
\text { Docente hace } 24 \text { años }\end{array}$ \\
\hline $\begin{array}{l}\text { Docente } 6 \\
\text {-Magister en educación virtual } \\
\text { - Licenciada en Educación } \\
\text {-Tiempo de dedicación: Completo } \\
\text {-Tiempo en la institución: } 4 \text { años }\end{array}$ & $\begin{array}{l}\text { Magister en Educación Virtual de la universidad cooperativa de } \\
\text { Colombia } \\
\text { Licenciada en educación de la Universidad Santander - Udes. } \\
\text { Docente hace } 9 \text { años }\end{array}$ \\
\hline
\end{tabular}

Por otro lado, la investigación establece cuatro categorías que se relacionan entre sí, categoría 1: metodologías educativas docentes, categoría 2: herramientas tecnológicas, categoría 3: didáctica de la tecnología educativa, categoría 4: competencias Digitales.

\begin{tabular}{|c|c|c|c|}
\hline $\begin{array}{l}\text { Objetivos (Especi- } \\
\text { ficos) }\end{array}$ & Categorias & Subcategorias & Instrumentos \\
\hline $\begin{array}{l}\text { Identificar cuáles } \\
\text { son las metodolo- } \\
\text { gías utilizadas por } \\
\text { los docentes en el } \\
\text { proceso de ense- } \\
\text { ñanza. }\end{array}$ & $\begin{array}{l}\text { Metodologías edu- } \\
\text { cativas docentes }\end{array}$ & $\begin{array}{l}\text { Aprendizaje basado en proyectos } \\
\text { Aprendizaje colaborativo } \\
\text { Gamificación } \\
\text { Aprendizaje basado en problemas } \\
\text { Design Thinking } \\
\text { Aprendizaje Basado en el Pensamien- } \\
\text { to } \\
\text { Aprendizaje Basado en Competencias }\end{array}$ & $\begin{array}{l}\text { Entrevista semies- } \\
\text { tructurada a docen- } \\
\text { tes }\end{array}$ \\
\hline
\end{tabular}




\section{Tabla 3 Matriz categorial}

\begin{tabular}{|c|c|c|c|}
\hline $\begin{array}{l}\text { Objetivos (Especi- } \\
\text { ficos) }\end{array}$ & Categorias & Subcategorias & Instrumentos \\
\hline $\begin{array}{l}\text { Examinar las carac- } \\
\text { terísticas asociadas } \\
\text { a las herramientas } \\
\text { tecnológicas em- } \\
\text { pleada en el área } \\
\text { de tecnología e in- } \\
\text { formática. }\end{array}$ & $\begin{array}{l}\text { Herramientas tec- } \\
\text { nológicas }\end{array}$ & $\begin{array}{l}\text { Redes entre pares } \\
\text { Efectividad de las Tic } \\
\text { Aprendizaje colaborativo } \\
\text { Aprendizaje colaborativo con soporte } \\
\text { TIC }\end{array}$ & $\begin{array}{l}\text { Encuesta Cuestiona- } \\
\text { rio de Observación }\end{array}$ \\
\hline \multirow{2}{*}{$\begin{array}{l}\text { Sugerir estrategias } \\
\text { didácticas o peda- } \\
\text { gógicas que forta- } \\
\text { lezca la metodolo- } \\
\text { gía utilizada por los } \\
\text { docentes del área } \\
\text { de informática en } \\
\text { el proceso de ense- } \\
\text { ñanza }\end{array}$} & $\begin{array}{l}\text { Didáctica de la tec- } \\
\text { nología educativa }\end{array}$ & $\begin{array}{l}\text { Teoría y práctica del proceso de ense- } \\
\text { ñanza- aprendizaje. } \\
\text { Concreción del currículo. } \\
\text { Programación en el aula: la unidad } \\
\text { didáctica. Selección y elaboración de } \\
\text { materiales educativos. }\end{array}$ & \multirow[t]{2}{*}{$\begin{array}{l}\text { Entrevista a docentes } \\
\text { y revisión documen- } \\
\text { tal }\end{array}$} \\
\hline & $\begin{array}{l}\text { Competencias digi- } \\
\text { tales }\end{array}$ & $\begin{array}{l}\text { Fomentar el aprendizaje. } \\
\text { Criterios de evaluación. } \\
\text { Organización de los espacios, de los re- } \\
\text { cursos y del tiempo. }\end{array}$ & \\
\hline
\end{tabular}

\section{RESULTADOS, ANÁLISIS Y DISCUSIÓN}

A continuación, se resume los hallazgos obtenidos con la aplicación del formato de observación no participante, la entrevista semiestructurada y el cuestionario docente para la categoría de metodologías educativas docentes.

Tabla 4. Triangulación de la información de la categoria metodologias educativas docentes

\begin{tabular}{|c|c|c|}
\hline \multicolumn{3}{|c|}{$\begin{array}{l}\text { Metodologias Educativas Docentes } \\
\text { Objetivo especiffico: Identificar cuáles son las metodologias utilizadas por los docentes en el proceso de enseñanza. } \\
\text { Subcategorias: Aprendizaje basado en proyectos, Aprendizaje colaborativo, Gamificación, Aprendizaje basado en } \\
\text { problemas, Design Thinking, Aprendizaje Basado en el Pensamiento, Aprendizaje Basado en Competencias. }\end{array}$} \\
\hline Subcategorias & Observación a publicaciones & Entrevista a profesores \\
\hline $\begin{array}{l}\text { Aprendizaje } \\
\text { basado en pro- } \\
\text { yectos }\end{array}$ & $\begin{array}{l}\text { En las publicaciones indagadas para esta investi- } \\
\text { gación se identifica que la educación actual está } \\
\text { enfocada a un aprendizaje basado en la coopera- } \\
\text { ción entre pares y las prácticas pedagógicas que } \\
\text { se desarrollan, evidenciando trabajo en grupo } \\
\text { basado en proyectos. Los docentes enfatizan en } \\
\text { las publicaciones como el trabajo colaborativo } \\
\text { actualmente es esencial para avanzar a nivel } \\
\text { educativo y especialmente con la aplicación de } \\
\text { herramientas educativas que fortalecen las acti- } \\
\text { vidades en las aulas de clase. }\end{array}$ & $\begin{array}{l}\text { Los docentes señalan la importancia del apren- } \\
\text { dizaje basado en proyectos (85,7\%) como una } \\
\text { metodología educativa necesaria para im- } \\
\text { plementar en los cursos no solamente por la } \\
\text { efectividad, sino además por la cantidad de es- } \\
\text { tudiantes que se orientan en las aulas de clase } \\
\text { ( } 40 \text { a } 50 \text { estudiantes por grado). } \\
\text { El docente debe estar en constante actualiza- } \\
\text { ción en cuanto a las herramientas tecnológicas } \\
\text { (plataformas, Aplicaciones y app interactivas), } \\
\text { para poder orientar y cumplir con las expecta- } \\
\text { tivas. }\end{array}$ \\
\hline
\end{tabular}

Fuente: elaboración propia. 
Tabla 4. Triangulación de la información de la categoría metodologias educativas docentes

\section{Metodologias Educativas Docentes}

Objetivo especifico: Identificar cuáles son las metodologías utilizadas por los docentes en el proceso de enseñanza. Subcategorias: Aprendizaje basado en proyectos, Aprendizaje colaborativo, Gamificación, Aprendizaje basado en problemas, Design Thinking, Aprendizaje Basado en el Pensamiento, Aprendizaje Basado en Competencias.

\begin{tabular}{|l|l|l|}
\hline \multicolumn{1}{|l|}{ Subcategorias } & \multicolumn{1}{|c|}{ Observación a publicaciones } & \multicolumn{1}{c}{ Entrevista a profesores } \\
\begin{tabular}{|l|l|l} 
Aprendizaje co- \\
laborativo
\end{tabular} & $\begin{array}{l}\text { Las investigaciones desarrolladas a nivel tec- } \\
\text { nológico concuerdan en la importancia del } \\
\text { trabajo colaborativo en el aula de clase y en- } \\
\text { tre pares docentes. }\end{array}$ & $\begin{array}{l}\text { El aprendizaje en las instituciones educa- } \\
\text { tivas y especialmente en las de carácter } \\
\text { público, están sujetas a este aprendizaje } \\
\text { (57,1 \%), por el número de estudiantes en } \\
\text { cada aula. }\end{array}$
\end{tabular}

Fuente: elaboración propia.

\section{Tabla 5. Triangulación de la información de la categoria herramientas tecnológicas}

\begin{tabular}{|c|c|c|}
\hline $\begin{array}{l}\text { tecnología e inf } \\
\text { Subcategorias: } \\
\text { borativo con so }\end{array}$ & Examinar las característic & $\begin{array}{l}\text { as } \\
\text { netodología empleada en el área de } \\
\text { dizaje colaborativo - Aprendizaje cola- }\end{array}$ \\
\hline Subcategorias & Observación a publicaciones & Entrevista a profesores \\
\hline $\begin{array}{l}\text { Efectividad de } \\
\text { las Tic }(71,4 \%)\end{array}$ & $\begin{array}{l}\text { En las investigaciones realizadas a partir del } \\
\text { año } 2000 \text { se evidencia la importancia de la } \\
\text { implementación de las Tic, donde ha ido } \\
\text { avanzando desde las Tic } 1.0 \text { hasta la actua- } \\
\text { lidad en } 3.0 \text {, estos avances han determina- } \\
\text { do un giro fundamental, evidenciándose la } \\
\text { constante participación de los docentes. }\end{array}$ & $\begin{array}{l}\text { El avance tecnológico ha sido drástico a } \\
\text { nivel pedagógico, teniendo que imple- } \\
\text { mentar herramientas tecnológicas que } \\
\text { permitan el proceso educativo e ir de la } \\
\text { mano con el avance tecnológico y por ello } \\
\text { la efectividad de las Tic es primordial en } \\
\text { este acompañamiento como competen- } \\
\text { cias básicas. }\end{array}$ \\
\hline $\begin{array}{l}\text { Aprendizaje } \\
\text { colaborativo } \\
\text { con soporte TIC } \\
(71,4 \%)\end{array}$ & $\begin{array}{l}\text { Los docentes de las diferentes asignaturas } \\
\text { de alguna u otra manera han tenido que } \\
\text { fortalecer sus estrategias pedagógicas apo- } \\
\text { yadas en el soporte de las Tic, para garan- } \\
\text { tizar procesos pedagógicos positivos en las } \\
\text { aulas de clase, así se evidencia en los artí- } \\
\text { culos de revista que indagan sobre el tema } \\
\text { y que cada día estos contenidos están más } \\
\text { en la red }\end{array}$ & $\begin{array}{l}\text { En la actualidad en la "era tecnológica" } \\
\text { así llamada por varios investigadores, el } \\
\text { internet ha sido un impedimento para la } \\
\text { implementación de algunas comunida- } \\
\text { des educativas, ya que no cuenta con los } \\
\text { recursos necesarios para su implemen- } \\
\text { tación, pero donde se obliga de alguna } \\
\text { u otra manera seguir sigilosamente en el } \\
\text { proceso tecnológico. }\end{array}$ \\
\hline
\end{tabular}

Fuente: elaboración propia. 


\section{Tabla 6. Triangulación de la información de didáctica de la tecnología Educativa}

\begin{tabular}{|c|c|c|}
\hline $\begin{array}{l}\text { Objetivo especifice } \\
\text { da por los docentes } \\
\text { competencias digit } \\
\text { Subcategorias: Teo } \\
\text { gramación en el au } \\
\text { el aprendizaje - Crit }\end{array}$ & $\begin{array}{l}\text { Tercera categoria de ané } \\
\text { Didáctica de la tecnología Ec } \\
\text { Sugerir estrategias didácticas o pedagógic } \\
\text { del área de informática en el proceso de er } \\
\text { les. } \\
\text { a y práctica del proceso de enseñanza - ap } \\
\text { : la unidad didáctica - Selección y elaborac } \\
\text { rios de evaluación. }\end{array}$ & $\begin{array}{l}\text { s } \\
\text { que fortalezca la metodología utiliza- } \\
\text { nuanza para el fortalecimiento de las } \\
\text { dizaje - Concreción del curriculo - Pro- } \\
\text { de materiales educativos - Fomentar }\end{array}$ \\
\hline Subcategorias & Observación a publicaciones & Entrevista a profesores \\
\hline $\begin{array}{l}\text { Teoría y práctica del } \\
\text { proceso de ense- } \\
\text { ñanza - aprendizaje. }\end{array}$ & $\begin{array}{l}\text { En las investigaciones recogidas, este aná- } \\
\text { lisis de teoría y práctica del proceso de } \\
\text { enseñanza (71,4 \%) se evidencia que las } \\
\text { dos van de la mano en el proceso de en- } \\
\text { señanza, los docentes deben implementar } \\
\text { contenidos tradicionales (Teoría) como re- } \\
\text { quisito indispensable en la elaboración de } \\
\text { las clases dando cuenta de pasar de lo tra- } \\
\text { dicional a lo práctico. }\end{array}$ & $\begin{array}{l}\text { Los docentes de la institución deben } \\
\text { entregar contenidos teóricos y estructu- } \\
\text { rados a los diferentes grados, para que, } \\
\text { a partir de allí, puedan poner en mar- } \\
\text { cha los diferentes proyectos educativos } \\
\text { de manera didáctica, que cumpla con } \\
\text { la finalidad de reforzar la parte teórica } \\
\text { y como muestra final para la dependen- } \\
\text { cia del área. }\end{array}$ \\
\hline $\begin{array}{l}\text { Programación en el } \\
\text { aula: la unidad di- } \\
\text { dáctica. Selección y } \\
\text { elaboración de ma- } \\
\text { teriales educativos. }\end{array}$ & $\begin{array}{l}\text { Se evidencia que las unidades didácticas } \\
\text { (57,1 \%) son tradicionales y no están actua- } \\
\text { lizadas, sin embargo, al llevarlo al aula de } \\
\text { clase se correlaciona con otros objetivos } \\
\text { que permiten apoyar las estrategias de } \\
\text { clase }\end{array}$ & $\begin{array}{l}\text { Los docentes estructuran sus clases con } \\
\text { los contenidos entregados en la insti- } \\
\text { tución, sin embargo los redirecciona a } \\
\text { fortalecer procesos dinámicos que in- } \\
\text { duzcan a la reutilización de material re- } \\
\text { ciclable, desarrollando el pensamiento } \\
\text { lógico creativo y dinámico. }\end{array}$ \\
\hline $\begin{array}{l}\text { Organización de los } \\
\text { espacios, de los re- } \\
\text { cursos y del tiempo }\end{array}$ & $\begin{array}{l}\text { Se observa que en la organización de los } \\
\text { espacios, de los recursos y del tiempo } \\
(57,1 \%) \text { es fundamental para desarrollar la } \\
\text { clase, debido a que en tecnología básica- } \\
\text { mente se desarrolla dos horas cátedra a la } \\
\text { semana, tiempo reducido para implemen- } \\
\text { tar proyectos única y exclusivamente en el } \\
\text { aula, por ende estos recursos y tiempos de- } \\
\text { ben ser utilizados favorablemente para no } \\
\text { caer en la monotonía. }\end{array}$ & $\begin{array}{l}\text { Los docentes consideran que una de } \\
\text { las subcategorias para tener en cuenta } \\
\text { es la organización de recursos, espacios } \\
\text { y tiempos, debido a que al implemen- } \\
\text { tarlos correctamente se desarrollan pro- } \\
\text { cesos mentales, creativos y dinámicos } \\
\text { que hacen que las clases se conviertan } \\
\text { en amenas y significantes para los estu- } \\
\text { diantes. }\end{array}$ \\
\hline $\begin{array}{l}\text { Fomentar el apren- } \\
\text { dizaje }\end{array}$ & $\begin{array}{l}\text { Con relación a fomentar el aprendizaje } \\
\text { ( } 42,9 \%) \text {, todas las investigaciones señalan } \\
\text { que es fundamental desarrollar el aprendi- } \\
\text { zaje, pero que en la actualidad se debe te- } \\
\text { ner en cuenta las didácticas tecnológicas } \\
\text { como estrategia fundamental en el desa- } \\
\text { rrollo de las competencias. }\end{array}$ & $\begin{array}{l}\text { Se evidencia como los docentes im- } \\
\text { plementan diferentes metodologías y } \\
\text { didácticas que fortalecen el aprendiza- } \\
\text { je en el aula de clase y habilidades de } \\
\text { comunicación en los diferentes grupos } \\
\text { de estudiantes. }\end{array}$ \\
\hline $\begin{array}{l}\text { Criterios de evalua- } \\
\text { ción }\end{array}$ & $\begin{array}{l}\text { Aunque la mayoría de investigaciones con- } \\
\text { cuerdan que a evaluación debería ser solo } \\
\text { una herramienta para medir los conoci- } \\
\text { mientos de los estudiantes, también men- } \\
\text { ciona diversas maneras de realizarlo. }\end{array}$ & $\begin{array}{l}\text { Los docentes manifiestan que el de- } \\
\text { sarrollo de las evaluaciones permite } \\
\text { medir una parte del coeficiente de los } \\
\text { estudiantes, sin embargo no son instru- } \\
\text { mento que deberían ser utilizados en la } \\
\text { educación, debido a que minimizan el } \\
\text { pensamiento y coarta la creatividad. }\end{array}$ \\
\hline
\end{tabular}

Fuente: elaboración propia. 


\section{Tabla 7. Triangulación de la información de la categoria de Competencias Digitales}

\begin{tabular}{|c|c|c|}
\hline \multicolumn{3}{|c|}{$\begin{array}{l}\text { Cuarta categoria de análisis } \\
\text { Competencias Digitales } \\
\text { ias didácticas o pedagógicas que fortalezca la metodología utiliza- } \\
\text { mática en el proceso de enseñanza para el fortalecimiento de las } \\
\text { nuevas tecnologias -Compartir información y contenidos -Partici- } \\
\text { ción mediante canales digitales - Gestión de la identidad digital . } \\
\text { por las competencias que deben desarrollar los estudiantes en el } \\
\text { lfabetización -Comunicación y colaboración Creación de contenidos } \\
\text { problemas }\end{array}$} \\
\hline Subcategorias & Observación a publicaciones & Entrevista a profesores \\
\hline $\begin{array}{l}\text { Creación de conteni- } \\
\text { dos digitales }(71,4 \%)\end{array}$ & $\begin{array}{l}\text { Los contenidos de las publicaciones se } \\
\text { presentan en diferentes formatos, eviden- } \\
\text { ciando la importancia de la creación de } \\
\text { contenidos digitales como herramienta } \\
\text { fundamental para el desarrollo de las te- } \\
\text { máticas. } \\
\text { Se evidencia la actualización y conocimien- } \\
\text { to por parte de los docentes que publican } \\
\text { contenidos a cerca de los contenidos digi- } \\
\text { tales. } \\
\text { Se evidencia la evolución que ha tenido las } \\
\text { investigaciones sobre el tema y dejan ver la } \\
\text { importancia de la creación de los mismos. }\end{array}$ & $\begin{array}{l}\text { Fomenta la creación de contenidos } \\
\text { digitales a partir de herramientas im- } \\
\text { ples gratuitas que se encuentran en la } \\
\text { red } \\
\text { Fortalece las competencias digitales } \\
\text { a través de diferentes procesos de } \\
\text { creación de aplicaciones, software } \\
\text { y demás recursos tecnológicos que } \\
\text { aportan al desarrollo pedagógico de } \\
\text { la clase. } \\
\text { Se desarrolla procesos formativos en- } \\
\text { tre pares, contribuyendo a la actuali- } \\
\text { zación y fortalecimiento en los cono- } \\
\text { cimientos de diferentes docentes }\end{array}$ \\
\hline $\begin{array}{l}\text { Resolución de pro- } \\
\text { blemas }(71,4 \%)\end{array}$ & $\begin{array}{l}\text { A la par con la subcategoría anterior, la re- } \\
\text { solución de problemas enmarca una serie } \\
\text { de estrategias pedagógicas que fortalecen } \\
\text { las competencias digitales en los estudian- } \\
\text { tes y clarifican las diferentes temáticas de } \\
\text { clase, siendo este un porcentaje alto en el } \\
\text { desarrollo de varias investigaciones curricu- } \\
\text { lares y científicas. }\end{array}$ & $\begin{array}{l}\text { La resolución de problemas en el pro- } \\
\text { ceso educativo se ha convertido en el } \\
\text { bum de la educación y no solo se im- } \\
\text { plemente en las clases de tecnología } \\
\text { e informática sino también en las dife- } \\
\text { rentes áreas, debido a la aplicabilidad } \\
\text { que tiene en el desenvolvimiento co- } \\
\text { tidiano de los estudiantes, permitien- } \\
\text { do generar de esta manera un pensa- } \\
\text { miento crítico, analítico y lógico. }\end{array}$ \\
\hline
\end{tabular}

Fuente: elaboración propia.

Una vez realizados los análisis a los diferentes resultados de esta investigación obtenidos de los instrumentos de recolección de datos utilizados para tal fin y aplicado a varios docentes de tecnología e informática, se puede mencionar los principales hallazgos encontrados en esta investigación.

- Los docentes desarrollan actividades que complementan las diversas temáticas, sin embargo, sus metodologías no fortalecen las competencias tecnológicas.

- Se evidencia que se desarrollan actividades en el aula de clase guiadas a conocer las diferentes teorías que abarca el plan de estudios.

- A pesar de tener la alianza con el Servicio Nacional de Aprendizaje, (Sena) dentro de 
la institución, las actividades que se desarrollan solo son de copiar en el cuaderno y responder preguntas acerca del tema que están aprendiendo, sin ser transversalizadas con las demás áreas de aprendizaje.

- Las actividades del Servicio Nacional de Aprendizaje (Sena), no está dirigido al fortalecimiento de las competencias digitales, por el contrario, aunque se hable de una formación integral, las metodologías siguen siendo tradicionales, no están realmente relacionados con la caracterización de los ciclos.

- Para los estudiantes que están inscritos en las actividades del Sena, no significa más que una nota dentro del proceso, es decir una serie de acciones con las que se deben cumplir porque son nota para una o varias asignaturas.

- Existe un espacio dentro de la jornada académica para fomentar las competencias digitales, sin embargo, son muy pocos los estudiantes que asisten, debido a que esta formación es opcional.

- Los docentes ya se adaptaron simplemente a chequear que se cumplan los indicadores mínimos, según lo establecido para cada proyecto y así garantizar el tema visto.

\section{CONCLUSIONES}

Las metodologías pedagógicas desarrolladas en el área de tecnología e informática, están muy relacionadas con diferentes métodos y estos a su vez a diferentes teorías del aprendizaje, permitiendo al docente interactuar con los estudiantes de diversas maneras, más aún, cuando los cambios que enfrenta la educación hoy en día, no solo por la crisis sanitaria que vive el país, sino a demás por el ritmo tan acelerado al que se enfrenta la juventud ante diferentes mecanismos tecnológicos, así lo exige. Y es desde allí, donde definitivamente, el docente debe hacer una retrospectiva para reflexionar sobre su labor docente y el servicio que presta a la sociedad actualmente como a futuro

Los resultados revelan que los docentes en general aplican métodos conductistas para asentar las bases necesarias a las temáticas planteadas dentro del aula de clase y poder de esta forma aplicar metodologías de acuerdo a las posibilidades de la comunidad que orienta, comunidad que como se menciona al principio de esta investigación, es una población de bajos recursos, donde en la mayoría de los casos, los padres de familia no hacen el acompañamiento adecuado a sus hijos, dejándolos a la deriva y a su vez trabajando con los pocos recursos que encuentran a su alrededor, factor que dificulta el trabajo docente en el aula de clase y por lo cual este termina con sus metodologías tradicionales y pragmáticas para poder alcanzar sus objetivos, sin concernir la importancia que tenga en los estudiantes a nivel social o personal.

No obstante, es importante aclarar que de acuerdo con la crisis sanitaria derivada por la COVID-19, los docentes tuvieron que enfrentarse a metodologías activas, capaces no solo de fortalecer las competencias digitales en los estudiantes, sino que además se convirtieron en la única metodología que se puede emplear, dejando más que claro, que los docentes de tecnología e informática, están capacitados para emplear cualquier herramienta tecnológica 
que apoya estas competencias, pero que en el aula no son tan importantes en el momento de la enseñanza-aprendizaje.

\section{REFERENCIAS}

Alonso, M. (2017). ¿Hacia una nueva definición de utilidad del conocimiento científico? Primer análisis de los proyectos de desarrollo tecnológico y social (PDTS) en Argentina. Trilogía Ciencia Tecnología Sociedad, 9(17), 79-97. https://doi.org/10.22430/21457778.626

British Council. (2018). La jornada. Niñas de AL crean prototipos tecnológicos en un programa auspiciado por Gran Bretaña. Recuperado de https://www.jornada.com. $\mathrm{mx} / 2018 / 11 / 22 /$ ciencias/aO2n1cie

Bunge, M.(2017). Educación y ciencias tecnológicas. http://wwwandrese.blogspot. com/2017/11/la-tecnología-según-varios-autores.html

Campos, G. (2017). La alfabetización tecnológica: de la informática al desarrollo de competencias tecnológicas. Universidad Astral de Chile.

Castilla, H.(2018). Más allá de los conocimientos. El arte de ser profesor mediador en el proceso de aprendizaje. Bogotá: UNIMINUTO

Dashner (2015). ¿Tecnología al servicio de la humanidad? o ¿humanidad al servicio de la tecnología? Un asunto sin resolver. https://disertacionessobreliteratura.wordpress. $\mathrm{com} / 2015 / 10 /$

Giner, S. (2008). Tecnocultura, saber y mudanza social. http://www.quadernsdigitals.net/ datos/hemeroteca/r 32/nr 331/a 4208/4208.html el 5 de octubre de 2015.

Guevara, E. (2017). Prototipos tecnológicos exitosos e innovadores de bajo presupuesto realizados por los alumnos en universidades públicas. Revista Electrónica ANFEl. https:// www.anfei.mx/revista/index.php/revista/article/view/323/964

Hernández, R. (2017). Impacto de las TIC en la educación: Retos y Perspectivas. Propósitos y Representaciones, 5(1), 325 - 347 http://dx.doi.org/10.20511/pyr2017.v5n1.149

Martínez, C. (2011). El método de estudio de caso, estrategia metodológica de la investigación científica. Revista Cientifica Pensamiento y Gestión.

Ocaña, Y., Valenzuela, L. y Morillo, J. (2020). La competencia digital en el docente universitario. Propósitos y Representaciones, 8(1), e455. doi: http://dx.doi.org/10.20511/ pyr2020.v8n1.455 